\title{
Minding a Healthy Body: Clarifying Media Roles as Primers in the Rating of Body Satisfaction in a Variety of Social Categories
}

\author{
Sebastian $\mathrm{G}^{*}$ \\ Department of Psychology, Scotland \\ *Corresponding author: Sebastian G, Department of Psychology, Scotland
}

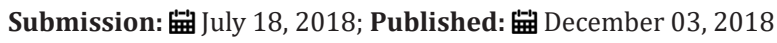

\begin{abstract}
In current literature surrounding body image and media, there is an overwhelming sense of the responsibility of media as a means of skewing expectations of beauty. This skewing process has been explained by a multitude of theories, however the Social Comparison Theory and the Tripartite Model generate an explanation by both the means and the value of media functioning as a prime in the perception of body image. However, in contemporary studies, findings challenging this this responsibility have emerged.

This study attempts to clarify the immediate role that media plays in the processes which generate body image satisfaction in individuals. This is done via chi-square tests for independence on the 360 questionnaire participants responses regarding their pre-priming body satisfaction, prime category (Overweight, Underweight and Healthy Eating), post priming body satisfaction and the socially descriptive categories of participants. These results indicate that there is observed relationship between media inspired primes and the body satisfaction rating of participants. When compared toward social categories, only individuals diagnosed with a Body Image Disorder demonstrate a significant relationship with priming and changing body satisfaction ratings. The implications of these findings to existing literature is discussed and limitations are addressed.
\end{abstract}

Keywords: Social comparison theory; Tripartite model; Body image; Body satisfaction; Media; Priming

\section{Introduction}

Humans are socially informed creatures. Their lives as well as their ambitions all pivot around factors meticulously investigated throughout the course of scientific history. The scientific field of social psychology has been of particular zeal in this pursuit. It was in the mid-twentieth-century that prominent explanations of social evaluations of the self, began to really influence the scientific community. As an example, the 1954 Social Comparison Theory by Leon Festinger helped build the foundations which today influence the structure for what we call social comparison. It is this scientific platform which allows modern research into both selfperception and influences from external sources [1]. This study attempts to expand on existing knowledge surrounding self-body satisfaction and perceptions of female body images by determining susceptibility of priming on different social categories of in the form of media

This study has selected the body satisfactions individuals' rate towards their own body as the means of identify priming from media. Several theories and studies attempt to explain concretely what body satisfaction is, but this study has selected the following explanations. Body Satisfaction refers to the self-perception individuals have, on a positive-negative continuum, towards their own body (citation). This varies on very unhappy, to very happy, and represents the polar nature of appraisals as explained by
Buunk et al. [2]. This spectrum is thought to exist due to exist as a result of individuals' self-appraising their body weight as socially informed, from severely underweight, to ideal, to average and up to severely overweight [3].

By investigating the dual process of self and socially informed appraisals in the context of priming, the evaluation of extraneous factors on this process can be identified in a contemporary context. In order to successfully do this, structured theories of the social comparison and media must be deployed. There mentioned 1954 Social Comparison Theory is one such theory particularly relevant to this rationale. Leon Festinger, the currently renowned social psychologist, developed Social Comparison Theory in order to explain the social comparison process that individuals engage in, and the dimensions at work within such comparisons. Notably, this theory conceptualized that comparative behaviors of individuals are done in order to clarify their self-perception of themselves [4]. This self-perception constitutes a relative identity towards a comparison of existing members of a social group and the individual, producing a ranking on a societal spectrum of appraisal [5]. This appraisal then generates the societal identity of the individual and dictates the negative or positive category of the individual [6].

In the context of body satisfaction and findings from the [7]. Participants were observed to generate individual identities from 
comparison. Participants generating more bodyweight altering behaviors, such as attending fitness programs and increase the likelihood of generating psychological disorders relating to body weight [7]. The Social Comparison Theory would suggest that this is takes place due to more skewed comparisons occurring as a result of more opportunities for socially comparing individual to societal body images [5]. These effects can both be explained by the increase in media's roles in our lives and contemporary increases in exposure to unhealthy standards of beauty [8].

This interpretation of [7] relies on media functioning as a means of priming. By media being a means of accessing societal information to inform the social comparative appraisal process individuals use to determine their body satisfaction, media functions as a prime $[5,7,8]$. Whether it involves displaying images of individuals, specific facial expressions or if it is the provision of academic evidence and observed impact on primes in the form of media have been well established in generating powerful influence on the judgments made by participants $[9,10]$. This relational connection has been explored in the formation of the Tripartite Influence Model [1]. This model was based on an original study conducted by Heinberg et al [11] Peer comparison and media images were identified as the two largest factors influencing self-perception and informed judgments on body dissatisfaction. Utilizing these findings, The Tripartite Influence Model suggests that the existing relationship between peer and family environment, media and body dissatisfaction are all most relevant to the body comparison tendencies of individuals. This would suggest, and in fact support the claim, that media functions as a form of priming experienced by individuals which can facilitates social comparisons on body satisfaction.

Priming is the process of cognitively predisposing individuals towards a desired response by presenting them with semantically relevant materials [12]. Higher frequencies of comparisons to more abnormal weights would predispose skewed personal perception rankings and would therefore elevate risks of body image disorders, eating disorders and the increase in weight loss program $[5,7,8,13]$. As Media is theorized to represent a mundane priming process, the presence of media must communicate impressions or indirect knowledge to influence individuals [14].

This priming can vary from traditional adverts containing images of specific body types, or articles informing about the nature of health in the population [14-17]. Regardless of the form, the role of the media remains constant: an external information source that the social comparative process of the individuals utilizes [1]. For this reason's media represents, in the context of both the Social Comparison Theory and the Tripartite Influence Model, the most direct and experimentally manipulatable means of influencing body satisfaction using priming [1,5] It is this particular quality which lends itself so excellently to the ambitions of this study. By manipulating primes in the form of regular media formats, this study should be able to determine the impact external sources have on the aforementioned process of satisfaction judgments.

Although media, from the provided theoretical frameworks, does represent an ideal candidate to generate an influential on in- dividual's body satisfaction, incongruent findings challenges the legitimacy of this claim [18]. Most notably, the findings of positive influences on body satisfaction as a result of negative priming from media [19-21]. Priming stimulus taking the form of media presenting thin females images generated higher levels of body satisfaction in individuals with higher Conscientiousness and traits associated with higher executive functioning [18].

This evidence suggests that there may possibly be a mediating factor in the influence of priming as a result of personality traits and cognitive control. This information is relevant as it eludes to the non-directional nature in which body satisfaction may vary from media exposure [19-22]. This does not confound with the Social Comparison Theory or the Tripartite Model, as it simply suggests the potential of such comparisons and priming factors may vary in the extent in which they are cognitively applied to the individual's perceptions [1,5]. As a result, this study accepts these confounding studies in the general literature and offers their application to the aims of this study. If both negative and positive changes to body satisfaction has been recorded as a result of media priming, is it possible that there it is yet to be determined in priming itself is successful?

This study will attempt to clarify if the media as a prime is an active participant in this process by which individuals generate their body satisfaction. Determining. This will generate support or critique for the relevancy of both the Social Comparison Theory and the Tripartite Model in the context of body image. This will also generate data which will clarify the distinction between the directional influence of media on body satisfaction uncovered in existing literature. Further analyzing on the specific social categories participants belong to and the change of their body satisfaction resulting from priming will be done to identify more susceptible categories of participants to establish if other confounding traits generate these mixed findings. This study will conclude by evaluating what these results suggest in the context of wider societal categories and the influence of media on sensitive topics such as body image.

\section{Hypotheses}

A. It is expected that individuals will experience a change in their self-body satisfaction as a result of priming, regardless of the particular direction of the priming stimuli.

B. It is expected that that data analysed will show significant relationships between difference in body satisfaction rating resulting from priming and the categories individuals belong to.

\section{Methods \\ Participants}

The participants in this study numbered a total of $360,287 \mathrm{fe}-$ males and 73 males. These participants ranged in age from 18 to $45+$ and were categorized in the following age sets: 18-24, 25-34, $35-44$, to $45+$; however, the vast majority (59.4\%) were within the 18 - 24 category with $20 \%, 6.93 \%$ and $14.17 \%$ in the other age-sets respectively. They were collected via a combination of snowball and 
convenience sampling, mediated through social media platforms (such as Facebook) and participation was voluntary. This particular process meant no exclusion criteria other than being over the age of 18 was introduced. However, this criteria could not be entirely enforced due to the open sampling technique and the ability for participants to provide false information. Participants could abort participation at any time, with Each participant gave acknowledgement of willingness to participate and allowance of the use of their data through a consent form, which also explained that participation could be terminated at any given time. The experiment was approved by the University of Stirling Psychology Ethics Committee.

\section{Materials and Apparatus}

The study required participants to access the internet on some sort of device (phone, computer or tablet) in order to access the questionnaire. The questionnaire was a designed using Qualtrics, an online questionnaire generating software, and consisted of fixed response questions. An information sheet as to the project aims and thank you for volunteering was the initial component of the experiment. The second was a consent form, whereby individuals could agree or disagree to the terms involved in participating. This was followed by nine questions asking individuals to categorize themselves within specific social dimensions, with a range of responses available. These were:
a. Age
b. Gender
c. Marital status
d. Previous diagnosis of eating disorders
e. Previous diagnosis of body image disorders
f. Completion of $30+$ minutes exercise in a week
g. Participation in a weight loss programmed
h. Diet
i. Pre-priming body satisfaction

Priming of the participant followed from the questionnaire responses. This took the form of the participant reading one of three short paragraphs (assigned at random) containing specific facts. Two of the primers were directional in nature (underweight and overweight) and gave facts about the current societal situation on their specific topic. The third primer was more neutral, but still semantically relevant (healthy eating). Once exposed to one of the randomly selected primers, six stimuli of a computer-modeled female body image were individually presented at random. Each body image stimulus required a fixed-response task. These fixed responses took the form of a Likert Scale, with each response option being the correct classification of one of the generated stimulus:
A. Severely underweight- BMI 9.2-24.9 kilogram
B. Slightly underweight- BMI 15.9- 42.9 kilogram
C. Ideal weight- BMI 22.5- 60.8 kilogram

\section{Average weight - BMI 26.9- 72.8 kilogram \\ E. Slightly overweight- BMI 34.0- 91.9 kilogram \\ F. Severely overweight- BMI 46.1- 124.7 kilogram}

The six images were generated using BMI Visualizer (2017), a web program that generates body images from BMI. These body images possess no distinguishing features, such as faces, hair, clothing, and even skin tone, ensures the only identifiable variance between images are weight related measurements (thigh, waist, face and chest measurements) on the gray-skinned featureless body image. The computer-generated stimuli all represented a $165 \mathrm{~cm}$ tall female varying in BMI. Once the participants had been exposed to the priming and had completed scoring the six images, a repeat question on their body satisfaction was produced. Following this final question, the aims of the study and influence of priming was explained along with the contact information of the researchers and counselling support services through the questionnaire's final page.

\section{Procedure}

As this study's design utilized an online questionnaire, the procedure followed three specific phases. The first phase involved the generation of the Qualtrics questionnaire, and for it to be distributed via social media platforms as already mentioned. This process took place over ten days, where researchers and acquaintances made the questionnaire URL link availed. The second phase of the study was entirely dependent on participants completing the questionnaire. This structured completion process by participants has already been described in the above section. The final phase involved exporting the responses from participants from Qualtrics into SPSS and conducting the analysis process as described in the following section section.

\section{Analysis}

SPSS (version 23.0) was utilized in order to analyze the data. Data recorded was coded by age, gender, categorical dimension questions, pre-priming body satisfaction, prime condition, response to body image stimuli and post-priming body satisfaction. The independent variables (primes for underweight, overweight and healthy eating) were examined for their effects on the dependent variable (body satisfaction). Significance levels were set at $p=0.05$. We used chi-square tests for independence and cross tabulations in order to test for the relationship between the independent variables and dependent variable directly. Following that we split the data into prime groups, coded responses as a new categorical variable (change, no change), and compared pre-priming body satisfaction responses to this change variable via a chi-square test for independence. Finally, this coded variable was compared to participant's categories (Diagnosis of Body Image Disorder, Weight Loss Program Participation, Completion of 30+ Minutes Exercise a Week, Gender, Diet Health Rating, Diagnosis of an Eating Disorder, Marital Status and Age) using chi-square tests for independence to establish which social categories elicited significant influences of priming. 


\section{Ethics}

This study, as similar studies in this field, address topics which may have serious ethical considerations [23]. Any time body image is investigated, it is crucial to acknowledge the nature of risk that it invoked. Initially extreme priming stimuli visually depicting anorexic or morbidly obese individuals was conceptualized for the study. An ethics committee rightfully rejected this suggestion due to the risks viewing such images would have on vulnerable individuals. For this reason, a less extreme redesign to priming was employed, where health statistics were substituted for the images as a means of priming. This reduced the risk of negatively influencing the psychological wellbeing of participants. As this did not guarantee the alleviation of such risks entirely, links to the Beating Eating Disorders and Anorexia \& Bulimia website's support forum was provided, as well as support pages for body image issues and obesity [23-26].

\section{Result}

\section{Priming influencing body satisfaction}

Running of a two-tailed chi-square test between the pre and post priming rating of body satisfaction in all participants, regard- less of priming group, yielded no significant evidence to reject the null hypothesis, $\mathrm{X}_{2}(4, \mathrm{~N}=360)=7.52, \mathrm{P}>0.111$ (Figure 1$)$. There was no relationship between priming as a whole and any change in the ratings of self-body satisfaction by participants. Only $14.2 \%$ of the 360 individuals changed their scores between pre and post priming $(\mathrm{N}=51)$, with the increase and decrease of satisfaction being split $6.4 \%$ and $7.8 \%$ respectively amongst the change population. For these reasons' directional relationship between priming and satisfaction change was not supported.

Upon splitting results by prime group and performing a Twotailed chi-square test on the relationship between pre-prime responses and the newly coded change in body satisfaction variable, we could identify the change occurring within specific primes. Participants primed with overweight primes generated a similar finding to the initial finding: $\mathrm{X}_{2}(4, \mathrm{~N}=132)=7.54, \mathrm{P}>0.110$ (Figure 1). Underweight priming was relatively worse, $X_{2}(4, N=115)$ $=4.609, P>0.330$, and healthy eating priming concrete insignificant findings: $\mathrm{X}_{2}(4, \mathrm{~N}=113)=2.75 \mathrm{P}>0.601$ (Figure 1). (Left to Right) The $\mathrm{X}_{2}$ Value generated for All Primes, Overweight Prime, Underweight Prime and Health Eating Prime towards directional change in pre to post priming body satisfaction rating, against the Critical Value at 4 degrees of Freedom at 0.05 significance level.

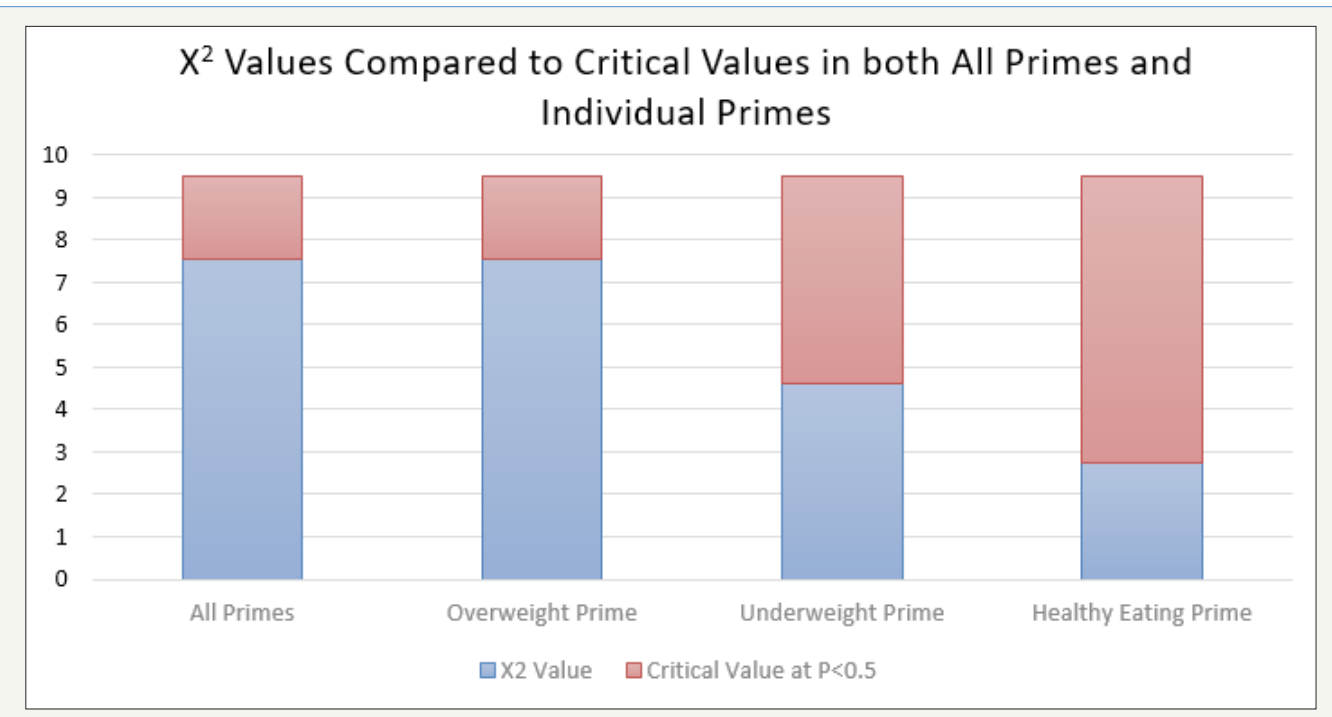

Figure 1: X-Axis: Prime categories.

Y-Axis: Numerical value.

\section{Social categories on change in body satisfaction}

When dividing the study sample into its respective categorical data only a single category successfully generated a significant finding. Participants which had been diagnosed with a Body Image Disorder showed a significant likelihood of changing their body satisfaction after priming $\mathrm{X}_{2}(2, \mathrm{~N}=360)=7.72, \mathrm{P}<0.005$ (Figure 2). Bootstrapping confirmed these results, suggesting the effects are robust. No other significant effects of priming were found. When participants participated in a weight loss program there was a close, but still insignificant association with changing body satisfaction after priming: $\mathrm{X}_{2}(1, \mathrm{~N}=360)=3.01, \mathrm{P}>0.083$ (Figure 2). Completion of $30+$ minutes yielded further insignificant findings:
$X_{2}(3, N=360)=4.60, P>0.203$, just as gender did: $X_{2}(1, N=360)$ $=0.787,7, \mathrm{P}>0.379$ (Figure 2 ).

Participants diet categories showed worse insignificance X2 $(4, \mathrm{~N}=360)=2.52, \mathrm{P}>0.641$ (Figure 2). Interestingly participants' diagnosis of possessing an Eating Disorder also showed no response to priming, $\mathrm{X}_{2}(1, \mathrm{~N}=360)=0.16, \mathrm{P}>0.690$ (Figure 2). Neither marital status $X_{2}(5, N=360)=2.76, P>0.737$, nor age were associated with changes in body satisfaction between pre and post priming: $X_{2}(3$, $\mathrm{N}=360$ ) $=0.86, \mathrm{P}>0.834$ (Figure 2). (Left to Right) The $X_{2}$ Value generated for Body Image Disorder, Weight Loss Program, Exercise, Gender, Diet, Eating Disorder, Marital Status and Age towards Change occurring between pre to post priming body satisfaction 
rating, against the Critical Value against respective degrees of Freedom at 0.05 significance level.

\section{Discussion}

This study produced results lacking sufficient support that priming on the subjects of body weight and healthy eating can influence an individual's body satisfaction (Figure 1). Further analysis into possible susceptibility of social categories found that the vast majority of variables investigated yielded no significant means of identifying a relationship between changing body satisfaction between pre and post priming responses (Figure 2). Only a single significant finding was identified. Diagnosis of a Body
Image Disorder indicated a susceptibility to change their body satisfaction ratings between pre and post priming, suggesting that a degree of vulnerability is present in these participants. Confounding this, and the wider body of literature surrounding body image and eating disorder, is Eating Disorder Diagnosis yielding no such finding [27]. These results indicate that priming within this study did not yield an observable influence on changing body satisfaction, but that individuals who changed their body satisfaction were significantly associated with participants and their diagnosis of Body Image Disorders, but not another form of weight associated disorder.

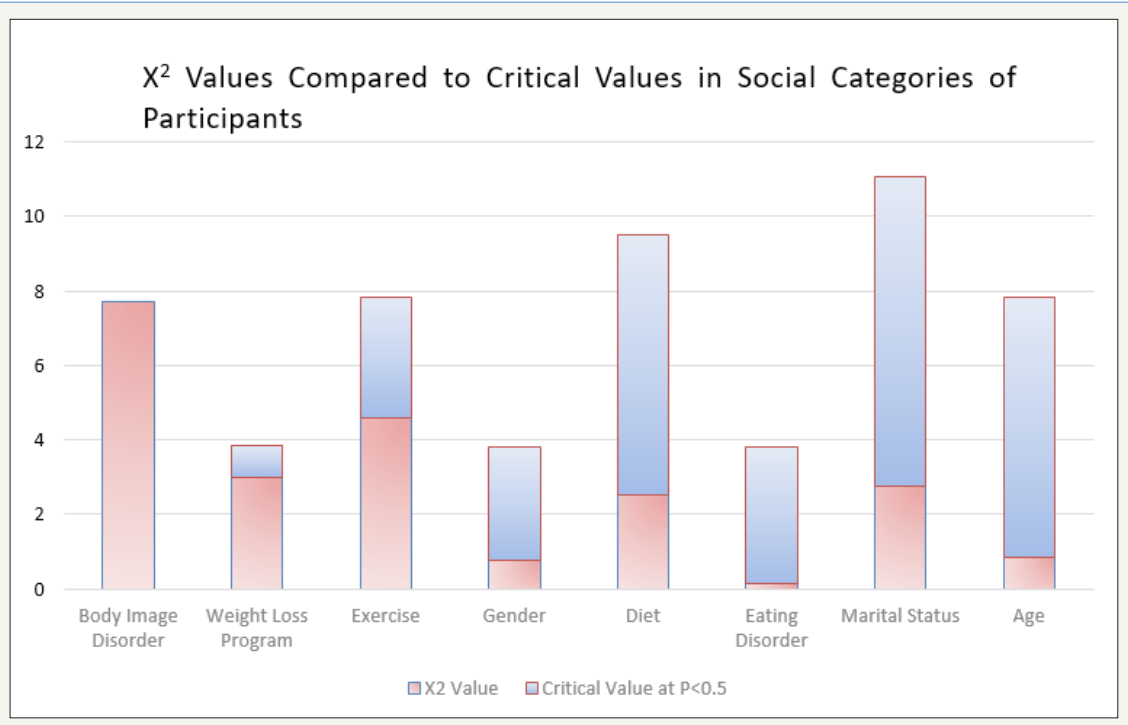

Figure 2: $X$-Axis: Participant social categories.

Y-Axis: Numerical value.

With an insignificant result when determining the effect of priming, the study must reject its first hypothesis and establish that there is in fact not an observed change in individuals' body satisfaction as a result of priming, regardless of the direction of the primes. Although this finding was not the immediately predicted results-a relationship would be established-it does not conflict with associated literature. An example of this is the aforementioned Roberts and Good's [18] study on media images and female body dissatisfaction. Although their study identified an association between media images and Neuroticism, a personality trait informed by the Five-Factor Model, they were also unable to concretely identify an influence of media exposure onto body dissatisfaction changes [18-28].

This compliments this study's finding that diagnosis of a Body Image Disorder elicited a significant finding. In a similar way to Roberts and Good's Neuroticism, this study also identified just a single trait associated with changing body satisfaction [18]. This is also the reason why this study's second hypothesis is partially accepted. A specific category (Body Image Diagnosis) demonstrated a susceptibility to priming in body satisfaction. Of further interest is the nature of this finding, and that of neuroticism found by Robert et al. [18]. According to [29] people diagnosed with Body Image Disorders score higher on the Five-Factor Model's Neuroticism compared to non-diagnosed individuals. This finding would suggest [18] finding informs this study's findings.

The Social Comparison Theory would suggest that these results stem from high neuroticism individuals having a higher disposition to compare themselves to others [5]. With a preexisting susceptibility to compare oneself to exposed media forms, logically, individuals would thereby self-evaluate more and give more opportunities to change their body satisfaction. When presented new information, this could result in a higher cognitive probability to elicit a comparison [30] suggest this particular process can be explained by open mindsets. Open mindsets represent the cognitive charge towards a semantic field in the meta-cognition of individuals, and their current working memory [30]. These cognitive processes suggest that more meta-cognitive compatible primes will produce more influential priming towards individuals' decision making. It is this model of priming which could explain how individuals diagnosed with Body Image Disorders, may in fact be utilizing the primer information more in post-priming body satisfaction rating.

Unfortunately, two particular theoretical counterparts challenge this explanation. Firstly, the assumption that neuroticism of the Body Image Disorders makes participants more susceptible to change body satisfaction should be mirrored in similar categories, 
such as Eating Disorders [31]. This study found no such similarity. This is of particularly relevance when Eating Disorder diagnosis is also linked to neuroticism [32]. This suggests something specific to Body Image Disorders generated the effect in this sample. Secondly, priming studies have determined that open mindsets work in a counter intuitive manner in terms of attentional detail [30-33]. The more modest a prime, the more it solidifies existing dispositions, and as primes become more extreme, the likelihood of altering opinions increases [30].

For that reason, primes portraying concerning facts on body weight should produce the most dissimilar mindsets from the non-diagnosed participants, but the most 'modest' information for diagnosed individuals [32]. If this were the case, the opposite effect would be observed in both Body Image Disorders and Eating Disorders participants. Body satisfactions would have been stable in diagnosed individuals and produced adjusted ratings in the wider sample. This was not the case, suggesting, as commented on by Berg et al. [4] that a more comprehensive theoretical understanding of the phenomenon of body image may be required.

Although this suggestion seems valid, the nature of this study's limitations may offer insight into these findings. The largest limitation of this study is the nature in which the design was administered. As the questionnaire design meant individuals completed the questionnaire in their own time, outside of an experimental setting, confounding variables could not be controlled for. Duration, distractions and comprehension during priming could not be accounted for, each of which capable of affecting the reliability of responses [12]. Similarly, it provided the Social Desirability Effect to occur, as participants had the potential to generate false information in order to generate social desirability. The repercussion of this limitation is that the validity of these responses could not be assured.

Another limitation is the quantity of participants within datasets. There were only 5 participants diagnosed with Body Image Disorders (out of 12) who changed their responses on body satisfaction, the remaining 7 did not. In comparison, Eating Disorder diagnosed participants had twice the sample size $(\mathrm{N}=26)$ and yielded much more insignificant results, despite the number of participants changing only being two more. With such small sample sizes, the validity of the results must be questioned. Coincidental changes in responses are given greater weight in statistical analysis and risk generating a Type 1 error. Given that the sampling technique involved snowball sampling, the potential for this category's sample size to be higher may have been possible if the study carried on its data collection phase for longer.

The final limitation of the study was the priming stimuli. The three primes originally entailed real visual representations of severely underweight and overweight individuals, and an average body size. Ethical procedures forced a substitution of these primes. The new primes took the form of factual paragraphs, mimicking the role of media articles. This would represent legitimate primes capable of influencing body satisfaction as explained by the Tripartite Model [1]. This study must acknowledge that this change was appropriate as it reduced the risk of exposing individuals to potentially traumatic images, and in the cases of diagnosed individuals, may either trigger, remind or exacerbate existing mental duress. Further studies within in this area should attempt to reproduce the experiment in an experimental setting where confounding variables can be controlled for, and direct assistance can be given, allowing the usage of more influential priming stimuli $[34,35]$.

As it stands, there are several dimensions which offer promising platforms for further study. The close-to-significance of individuals in a weight loss program may offer more information regarding the active active process of improving health on cognitive processing involved in interpreting priming [30]. Should an experiment be conducted on a control group of weight loss program participants and non-participants, findings may support or challenge existing ideas such as the Social Comparison model or findings from the $[5,7]$.

\section{Conclusion}

In conclusion, this study offers little support to a cohesive picture on what role priming via media has on body satisfaction. This study must reject its first hypothesis, that there is expected to be a change in self-body satisfaction as a result of priming, regardless of the particular direction of the priming stimuli. As a result, it can be said to support the incongruent studies of body image in contrast to the wider literature. Furthermore, this study may partially confirm its second hypothesis, that there is an expectation that data analyzed will show significant relationships between the difference in body satisfaction rating and the categories individuals belong to. This partial confirmation originates from a single identified category. Body Image Disorder diagnosis reflects a statistically significant relationship towards changing pre to post body satisfaction. There seems to be no other category offering susceptibility towards media priming and body satisfaction. This study concludes with addressing the complexity of investigating body satisfaction and priming in response the media and suggests that until a more experimentally controlled replication takes place, the use of this study in clarifying this relationship is irresponsible.

\section{References}

1. Thompson J, Heinberg L, Altabe M, Dunn ST (1999) Exacting beauty: Theory, assessment and treatment of body image disturbance. American Psychological Association, Washington, USA.

2. BuunkB, Collins R, TaylorS, Yperen NV (1990) The affective consequences of social comparison: Either direction has its ups and downs. Journal of Personality and Social Psychology 59(6): 1238-1249.

3. Cafri G, Thompson J, Ricciardelli L, Cabe MM, Smolak L, et al. (2005) Pursuit of the muscular ideal: Physical and psychological consequences and putative risk factors. Clinical Psychology Review 25(2): 215-239.

4. Berg P, Paxton S, Keery H, Wall M, Guo J, et al. (2007) Body dissatisfaction and body comparison with media images in male and females. Body Image 4(3): 257-268.

5. Festinger L (1954) A theory of social comparison processes. Human Relations 7(2): 117-140.

6. Paxton S, Eisenberg ME, Sztainer DN (2006) Prospective predictors of body dissatisfaction in adolescent girls and boys: A five-year longitudinal study. Developmental Psychology 42(5): 888-899. 
7. Fallon E, Hausenblas H (2005) Media images of the "ideal" female body: Can acute exercise moderate their psychological impact? Body Image 2(1): 62-73.

8. Stone E (2017) Sexy, Thin, and White: The Intersection of Sexualization, Body Type, and Race on Stereotypes about Women and Women 's Body Dissatisfaction. University of Kentucky, Unknowledge.

9. Irving LM (1990) Mirror Images: Effects of the standard of beauty on the self- and body-esteem of women exhibiting varying levels of bulimic symptoms. Journal of Social and Clinical Psychology 9(2): 230-242.

10. Stice E, Shaw H (1994) Adverse effects of media portrayed thin-ideal on women and linkages to bulimic symptomatology. Journal of Social and Clinical Psychology 13(3): 288-308.

11. Heinberg L, Thompson J (1992) Social Comparison: Gender, target importance ratings and relations to body image disturbance. Journal of Social Behavior and Personality 7: 335-344.

12. Carter DC (1997) Doing quantitative psychology research: from design to report/ David Clark-Carter. Hove: The Psychology Press, UK.

13. Muennig P, Haomiao J, Rufina L, Lubetkin E (2008) I think therefore i am: Perceived ideal weight as a determinant of health. American Journal of Public Health 98(3): 501-506.

14. Smith E (1998) Mental representation and memory. In: Gilberts D, Fiske S, Lindzey G (Eds.), The handbook of social psychology, McGraw-Hill, New York, USA, 1: 391-445.

15. Harrison K, Cantor J (1997) The relationship between media consumption and eating disorders. Journal of Communication 47(1): 40-67.

16. Mutz DC (1992) Mass media and the depoliticization of personal experience. American Journal of Political Science 36(2): 483-508.

17. Silverstein B, Perdue L, Peterson B, Vogel L, Fantini D (1986) Possible causes of the thin standard of bodily attractiveness for women International Journal of Eating Disorders 5(5): 907-916.

18. Roberts A, Good E (2010) Meda images and female body dissatisfaction: The moderating effects of the Five-Factor traits. Eating Behaviors 11(4): 211-216.

19. King EH, King DH (1997) Media effects on women's body esteem: Socia and individual difference factors. Journal of Applied Social Psychology 27(5): 399-417.

20. Joshi R, Herman P, Polivy J (2004) Self-enhancing effects of exposure to thin-body images. International Journal of Eating Disorders 35(3): 333341.

21. Mills J, Polivy J, Herman C, Tiggemann M (2002) Effects of exposure to thin media images: Evidence of self-enhancement among restrained eaters. Personality and Social Psychology Bulletin 28(12): 1687-1699.

22. Polivy J, Herman C (2004) Sociocultural idealization of thin female body shapes: An introduction to the special issue on body image and eating disorders. Journal of Social and Clinical Psychology23(1): 1-6.

23. Anorexia, Bulimia (2016) Anorexia and Bulima: How are they different?

24. https://www.berealcampaign.co.uk/resources

25. https://www.beateatingdisorders.org.uk/support-services/onlinegroups

26. https://www.dailystrength.org/

27. Hamilton K, Waller G (1993) Media influences on body size estimation in anorexia and bulimia: an experimental study. British Journal of Psychiatry 162: 837-840.

28. Costa P, Crae RM (1991) Facet scales for agreeableness and conscientiousness: A revision of the NEO personality inventory. Personality and Individual Differences 12(9): 887-898.

29. Podar I, Hannes A, Allik J (1999) Personality and affectivity characteristics associated with eating disorders: A comparison of eating disordered, weight-preoccupied and normal samples. J Pers Assess 73(1): 133-147.

30. Alexopoulos T, Fiedler K, Freytag P (2012) The impact of open and closed mindsets on evaluative priming. Cogn Emot 26(6): 978-994.

31. Dalley S, Buunk A, Umit T (2009) Female body dissatisfaction after exposure to overweight and thin media images: The role of body mass index and neuroticism. Personality and Individual Differences 47(1): 4751.

32. Claes L, Vandereycken W, Luyten P, Soenens B, Pieters G, et al. (2006) Personality prototypes in eating disorders based on the Big Five Model. J Pers Disord 2(4): 401-416.

33. Storbeck J, Clore G (2003) The affective regulation of cognitive priming. Emotion 8(2): 208-215.

34. Graw KM, Ling C (2003) Media priming of presidential and group evaluations. Political Communications 20(1): 23-40.

35. http://www.bmivisualizer.com/
Creative Commons Attribution 4.0 International License

For possible submissions Click Here
Submit Article

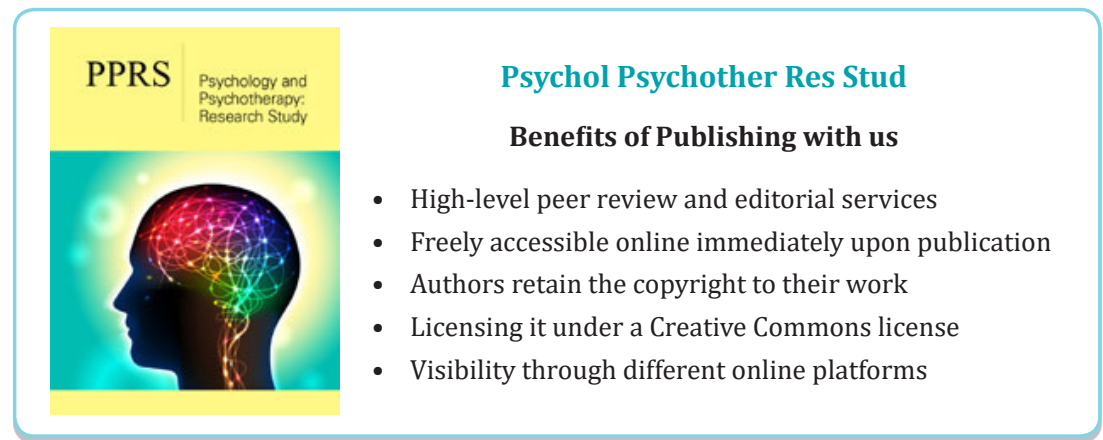

